\title{
Estado nutricional y resistencia aeróbica en escolares de educación básica: línea base de un Proyecto de Promoción de la Salud
}

\author{
Juliana Kain $\mathbf{B}^{\mathbf{1}}$, Sonia Olivares $\mathbf{C}^{\mathbf{1 b}}$, Marcela Romo $\mathbf{M}^{\mathbf{1 c}}$, \\ Bárbara Leyton $\mathrm{D}^{\mathbf{1 d}}$, Fernando Vio $\mathrm{D}^{\mathbf{1}}$, Ricardo Cerda $\mathrm{R}^{1} \mathrm{e}$, \\ Rosa González $\mathbf{P}^{2 f}$, Antonio Giadalah $\mathrm{A}^{3 \mathrm{~g}}$, Cecilia Albala $\mathbf{B}^{\mathbf{1}}$. \\ Nutritional status and aerobic \\ capacity among children attending \\ public elementary schools in Chile
}

Background: The Chilean Ministry of Health has developed a health promotion program called «ida Chile», whose aim is to target risk factors for chronic diseases. To achieve this, school-based initiatives in nutrition and physical activity have been implemented. The evaluation of these initiatives includes a baseline assessment of these two variables. Aim: Baseline assessment of the nutritional status and aerobic capacity of elementary school children (1st to 8th grade). Material and methods: Children from seven public schools were studied. Weight, height, triceps skinfold thickness and waist circumference were measured. To test the aerobic capacity, the 20-m Shuttle Run Test (Navette) was utilized. Results: The assessment included 4,271 children, mean age was 10.2 years. The percentile of body mass index, as compared to the CDC 2000 Reference was $67.8 \pm 26.7$ and $68 \pm 26.7$ for boys and girls, respectively. The prevalence of obesity among children from 6 to 8 years, was 20.3 and $17.7 \%$ for boys and girls, respectively. Approximately $30 \%$ of males and $15 \%$ of females with normal weight had a good aerobic resistance, compared to 15 and $4 \%$ of their overweight counterparts. Aerobic capacity decreased with increasing age. Conclusions: There is a high prevalence of obesity and a deficient level of aerobic capacity among children attending public schools in Chile (Rev Méd Chile 2004; 132: 1395-1402).

(Key Words: Aerobic exercise; Childhood eating and feeding disorders; Nutritional status; Obesity)

\footnotetext{
Recibido el 16 de abril, 2004. Aceptado en versión corregida el 10 de septiembre, 2004. Trabajo financiado por Chiledeportes, Ministerio de Educación y Córpora Tresmontes. ${ }^{1}$ Instituto de Nutrición y Tecnología de los Alimentos (INTA), Universidad de Chile. ${ }^{2}$ Ministerio de Educación. ${ }^{3}$ Chiledeportes.

aBioquímico, MPH. b Nutricionista, MSc. 'Antropóloga, MSc. dEstadístico. e Nutricionista, tesista Programa Magíster INTA. PProfesor de Educación Física, Magíster en Educación. gProfesor de Educación Física.
}

Correspondencia a: Juliana Kain. Av. Macul 5540, Santiago.

Fax: 56-2-2214030. E-mail: jkain@inta.cl 
Cosation on el propósito de responder a las demandas del actual perfil epidemiológico y demográfico del país, el Ministerio de Salud asumió el desafío, creando en 1998 el Consejo Nacional para la Promoción de la Salud, «ida Chile», entidad de carácter intersectorial que ha definido metas concretas al año 2010. Estas metas están relacionadas con aquellos factores de riesgo asociados a la calidad de vida de los chilenos, específicamente alimentación, sedentarismo, tabaquismo, factores psicosociales y ambientales. Para cumplir con las metas, las iniciativas en estas áreas están siendo implementadas en escuelas, lugares de trabajo y comunas ${ }^{1}$.

Los programas a nivel escolar, diseñados e implementados correctamente, pueden ejercer un rol muy importante en la promoción de la salud ${ }^{2}$. En ese sentido, la escuela promotora de salud representa una estrategia que pretende involucrar a toda la comunidad educativa, a través de la realización de actividades regulares en tres condicionantes en forma simultánea con al menos 50\% del alumnado. $\mathrm{Al}$ año 2002, alrededor de 620.000 escolares de todo el país se beneficiaron de esta estrategia ${ }^{3}$.

Para conocer la evolución de los factores de riesgo, y así poder estimar si las acciones logran una reducción de dichos factores, es necesario realizar una evaluación de impacto. La estrategia de escuela promotora de salud no contempla una evaluación de este tipo, información muy relevante para estimar la factibilidad de alcanzar las metas a 2010. En ese contexto, el año 2002, el INTA de la Universidad de Chile, con el apoyo técnico y financiero del Ministerio de Educación (MINEDUC), el Instituto Nacional del Deporte (Chiledeportes), el Ministerio de Salud (MINSAL) y la empresa Córpora Tresmontes, realizó un estudio diagnóstico de la situación nutricional, de actividad física y de educación ambiental en escolares de educación básica municipalizados, como asimismo determinó la factibilidad de que las escuelas seleccionadas sean «scuelas Libres de Humo de Tabaco». Posteriormente se desarrolló, implementó (durante 6 meses) y evaluó un proyecto piloto en promoción de la salud durante el año 2002, dirigido a escolares de educación básica municipalizada que incluyó cuatro componentes: alimentación, actividad física, tabaquismo y medio ambiente.

Este artículo muestra los resultados de la línea base relacionados con el estado nutricional y la resistencia aeróbica de los escolares, con el fin de diseñar y evaluar la implementación de estrategias en esas áreas.

\section{MATERIAL Y MÉTODO}

Este es un estudio descriptivo de corte transversal que contempló determinar en marzo/abril de 2002, el estado nutricional de escolares de educación básica y la resistencia aeróbica de aquellos entre $5^{0}$ y $8^{0}$ básico, como línea base para aplicar una estrategia de promoción de la salud.

Muestra. Esta incluyó a escolares de $1^{0}$ a $8^{0}$ básico de 7 escuelas básicas municipales, ubicadas en las ciudades de Santiago (La Florida), Curicó y Casablanca. Las escuelas fueron seleccionadas en conjunto con las autoridades municipales de acuerdo a los siguientes criterios de inclusión: escuelas básicas con jornada escolar completa, sin actividades previas ni actuales en alimentación/nutrición ni actividad física y con un índice de vulnerabilidad escolar (IVE) similar. El IVE de las escuelas (determinado por la Junta Nacional de Auxilio Escolar y Becas) fluctuó entre 34 y 42, lo que significa que el porcentaje de niños con necesidad de alimentación escolar variaba entre esas dos cifras. En la práctica, $62 \%$ de los niños eran beneficiarios del programa PAE (Programa de Alimentación Escolar).

La muestra total incluyó a 2.286 hombres y 1.985 mujeres, con un total de 4.271 escolares; el número de niños en cada categonía de edad fue similar, siendo la proporción de varones ligeramente superior. Por incluir esta muestra sólo niños hasta $8^{0}$ básico, el grupo sobre 14 años fue pequeño, motivo por el cual éstos fueron excluidos de algunos análisis.

Variables. Se registraron los siguientes datos en todos los niños:

a) Antropometría: se determinó peso, talla, perímetro de cintura y pliegue tricipital en todos los escolares de $1^{\circ}$ a 8 o básico. Esta evaluación fue realizada por nutricionistas, que fueron entrenados para minimizar tanto el error interindividual como intraindividual.

b) Aptitud física: profesores de educación física aplicaron la prueba de Navette $^{4}$ de resistencia aeróbica sólo a los niños entre $5^{\circ}$ y $8^{\circ}$, ya que se ha encontrado que en los niños menores, los resultados de esta prueba no son confiables ${ }^{5}$. 
Esta prueba consiste en registrar la velocidad máxima alcanzada por la persona cuando corre $20 \mathrm{~m}$ ida y vuelta a una velocidad creciente. La medición corresponde al último período (palier) en el cual se alcanza esa velocidad. Además, se determinó en los niños de $1^{\circ}$ a $8^{\circ}$, la prueba de Mathews (Informe de Trujillo y cols. Serie de Estudios № 241. CPEIP, MINEDUC, 1999) que mide flexibilidad de tronco. Para ello, el niño se para sobre una silla y se le solicita realizar la mayor flexión de tronco posible con los brazos estirados hacia abajo. Se registra la distancia entre la punta de los dedos y el asiento de la silla. Se seleccionaron estos componentes de la aptitud física por estar relacionados con la salud ${ }^{6}$ y por haber sido utilizados por investigadores de nuestro país país (datos obtenidos de Trujillo y cols, Montecinos y cols y Castillo y cols a partir de informes internos del Ministerio de Educación en 1999 y de las Universidades Católica del Maule y de Playa Ancha en 2001), lo que permite la comparación de resultados.

Estadística. Se calcularon los valores promedios (DE) de las variables antropométricas y de aptitud física en los escolares por sexo. Se determinó si había diferencias significativas por sexo en cada una de estas variables, utilizando el test estadístico T de Student. Se calculó el Indice de Masa Corporal $\left(\mathrm{IMC}=\mathrm{kg} / \mathrm{m}^{2}\right)$ para cada niño, lo que permitió determinar su percentil utilizando la referencia NCHS/CDC $2000^{7}$. La prevalencia de sobrepeso y de obesidad se determinó según la proporción de escolares con un percentil de IMC 85-95 y $\geq 95$ (según edad y sexo) de la referencia mencionada anteriormente, cuyo uso ha sido recomendado por la Unidad de Nutrición del MINSAL ${ }^{8}$. Se determinó si había diferencias significativas en la prevalencia de obesidad por sexo en las diferentes categorías de edad y dentro del mismo sexo por edad, utilizando el test de proporciones.

En relación a la prueba de Navette, se estimó pertinente comparar nuestros resultados con los que han obtenido otros investigadores nacionales. Esta incluyó niños a partir de los 10 años, ya que los estudios publicados fueron realizados con escolares mayores de esa edad. Se efectuó la comparación (promedios por edad y sexo) con dos estudios, el de Trujillo y cols ${ }^{4}$ realizado en 1998 con 548 escolares de 10 a 17 años de
Valparaíso y el de Castillo y cols ${ }^{9}$, efectuado en 1999 con escolares de 10 a 18 años de la V región. Se determinaron las diferencias por edad y sexo utilizando el test $\mathrm{T}$ para muestras independientes.

Se categorizaron los escolares según resistencia aeróbica, utilizando los puntos de corte propuestos por Montecinos y cols ${ }^{8}$, basados en la distribución percentilar obtenida de su estudio de 10.600 sujetos. Estos puntos de corte son: bueno, $\geq$ percentil (p) 60 , regular $<$ p $60 \mathrm{y} \geq \mathrm{p} 30$ y malo $<$ p 30. El p 60 corresponde a los siguientes valores en el caso de los hombres: 4, 5, 5 y 6 para $10,11,12$ y 13 años respectivamente, mientras que en las niñas éstos son: 4, 4, 4,5 y 5 .

A través de la prueba estadística de $\chi^{2}$, se determinó si había una asociación entre los resultados de las pruebas de Navette y Mathews y el estado nutricional de los niños.

\section{RESULTADOS}

En la Tabla 1 se presentan las características antropométricas y de aptitud física de los escolares de $1^{0}$ a $8^{\circ}$ básico por sexo. La edad promedio fue de 10,6 años en ambos sexos, mientras que el IMC fue casi igual, 19,3 y 19,6 en hombres y mujeres, respectivamente. Se encontraron diferencias significativas entre varones y niñas en el promedio de pliegue tricipital (mayor en las niñas) y perímetro de cintura (mayor en los varones). Se observó en ambos sexos que el promedio de percentil IMC fue elevado ( $p$ 68), 18

Tabla 1. C aracterísticas antropométricas y de aptitud física de escolares por sexo (prom \pm DE)

\begin{tabular}{|c|c|c|}
\hline & Hombres & Mujeres \\
\hline Edad (años) & $10,4 \pm 2,6$ & $10,3 \pm 2,5$ \\
\hline Peso (kg) & $38 \pm 13,1$ & $38 \pm 13,3$ \\
\hline Talla (cm) & $138 \pm 14,2$ & $137,1 \pm 14,1$ \\
\hline IMC & $19,3 \pm 3,6$ & $19,6 \pm 3,8$ \\
\hline Percentil IMC & $67,8 \pm 26,7$ & $68 \pm 26,7$ \\
\hline Pliegue Tric (mm) & $12,6 \pm 6,1$ & $15,1 \pm 6,1^{*}$ \\
\hline Perímetro cintura $(\mathrm{cm})$ & $66,4 \pm 10,4^{*}$ & $64,9 \pm 10$ \\
\hline Test de Mathews (cm) & $22,3 \pm 6,9$ & $23,5 \pm 6,8^{*}$ \\
\hline Test de Navette (períodos)a & $3,8 \pm 1,9 *$ & $2,7 \pm 1,2$ \\
\hline
\end{tabular}

*p $<0,05^{a}$ esta prueba fue aplicada en niños $>10$ años. 
puntos por sobre el percentil 50. Se encontraron diferencias significativas por sexo en ambas pruebas de aptitud física; la de flexibilidad (Mathews) fue mayor (mejor) en las niñas, en tanto la de resistencia aeróbica fue mayor en los varones.

En la Tabla 2 se presenta la proporción de niños con sobrepeso y obesidad por edad. El sobrepeso fluctuó en alrededor de $20 \%$ sin diferencias por edad o sexo. El porcentaje de obesos fue mayor en los niños menores, tanto en hombres como mujeres. No se encontraron diferencias significativas en el porcentaje de obesidad por sexo a ninguna edad.

La Tabla 3 muestra los valores promedios (DE) de la prueba de Navette por edad y sexo obtenidos en nuestro estudio, en el de Trujillo y cols y

\section{Tabla 2. Proporción de sobrepeso y obesidad por edad y sexo}

\begin{tabular}{|lcccc|}
\hline \multirow{2}{*}{ Edad (a) } & \multicolumn{2}{c|}{ Sobrepeso (\%) } & \multicolumn{2}{c|}{ Obesidad (\%) } \\
& H & M & H & M \\
\hline$<8$ & 20,8 & 20,2 & 20,3 & 17,7 \\
$\geq 8$ y $<10$ & 18,8 & 19,3 & 17,0 & 15,4 \\
$\geq 10$ y $<12$ & 20,8 & 20,7 & 16,5 & 14,0 \\
$\geq 12$ y $<14$ & 20,6 & 22,8 & 13,7 & 13,6 \\
\hline
\end{tabular}

$\chi^{2}=\mathrm{NS}$ en el de Castillo y cols. Al comparar nuestros datos con los del primer estudio, se observó que sus valores fueron significativamente mayores en los hombres mayores de 11 años y en las niñas mayores de 12 años. Al realizar la comparación con los resultados de Castillo y cols, se observó que sus cifras eran mejores en hombres, mientras que en mujeres, fueron prácticamente iguales.

No hubo una asociación entre el estado nutricional y flexibilidad ( $p=0,428$ y $p=0,166$ en hombres y mujeres, respectivamente).

Las Tablas 4 y 5 muestran la proporción de niños con estado nutricional normal y con sobrepeso (incluye obesos) en las 3 categorías de resistencia aeróbica: buena, regular y mala. En el caso de los varones con estado nutricional normal (Tabla 4), se observa que alrededor de $30 \%$ de ellos tiene una buena resistencia aeróbica, mientras que en aquellos con sobrepeso, hay sólo entre 9,4\% (10 años) y 18\% (12 años). La mayor proporción de los varones se ubica en la categoría mala», que fluctúa entre 40,5\% (10 años normales) y $77 \%$ en los de 12 años con sobrepeso. La Tabla 5 muestra la situación en las niñas. En las de estado nutricional normal, hay sólo entre 8,8\% (13 años) y 20\% (11 años) en la categoría buena, mientras que en las con sobrepeso estas cifras son significativamente más bajas, entre 2,7\% (10 y 11 años) y 5,7\% en las de 12 años. Al igual que en el caso de los hombres, la mayor parte de las niñas

Tabla 3. Comparación de resultados de prueba de $\mathrm{N}$ avette de tres estudios chilenos

\begin{tabular}{|lcccccc|}
\hline $\begin{array}{l}\text { Edad } \\
\text { y sexo }\end{array}$ & $\mathrm{N}_{1}$ & $\mathrm{~N}_{2}$ & $\mathrm{~N}_{3}$ & $\begin{array}{c}\text { Prom }_{1} \\
\pm \mathrm{DS}\end{array}$ & $\begin{array}{c}\text { Prom }_{2} \\
\pm \mathrm{DS}\end{array}$ & $\begin{array}{c}\text { Prom }_{3} \\
\pm \mathrm{DS}\end{array}$ \\
\hline $10 \mathrm{H}$ & 168 & 33 & 48 & $3,1 \pm 1,5$ & $2,9 \pm 1,7$ & $4,1 \pm 1,2^{*}$ \\
$10 \mathrm{M}$ & 155 & 32 & 40 & $2,4 \pm 0,97$ & $2,6 \pm 1,3$ & $2,5 \pm 1$ \\
$11 \mathrm{H}$ & 232 & 44 & 113 & $3,4 \pm 1,6$ & $4,4 \pm 1,7 *$ & $4,5 \pm 1,5^{*}$ \\
$11 \mathrm{M}$ & 181 & 41 & 47 & $2,7 \pm 1,2$ & $3,1 \pm 1,7$ & $2,5 \pm 1,1$ \\
$12 \mathrm{H}$ & 204 & 39 & 86 & $3,8 \pm 1,9$ & $5,3 \pm 2^{*}$ & $5,4 \pm 2,1^{*}$ \\
$12 \mathrm{M}$ & 196 & 31 & 42 & $2,8 \pm 1,2$ & $3,6 \pm 1,2^{*}$ & $2,7 \pm 1,1$ \\
$13 \mathrm{H}$ & 184 & 40 & 85 & $4,1 \pm 2,1$ & $5,1 \pm 2,3^{*}$ & $5,9 \pm 2,1^{*}$ \\
$13 \mathrm{M}$ & 179 & 34 & 56 & $2,8 \pm 1,3$ & $3,8 \pm 1,3^{*}$ & $2,3 \pm 1$ \\
\hline
\end{tabular}

${ }^{1}$ Estudio nuestro

${ }^{2}$ Trujillo y cols ${ }^{4}$

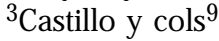

*Mayor que los resultados de nuestro estudio $\mathrm{p}<0,05$. 
tiene una mala resistencia aeróbica. En ambos sexos, se encontró una asociación entre estado nutricional y Navette en todos los grupos de edad, salvo en los de 13 años.

\section{DisCUSIÓN}

Una de las metas establecidas por Vida Chile para el año 2010 es la disminución de la obesidad en escolares de primer año básico de $17 \%$ en el año 2000 a $12 \%$ en el $2010^{1}$. También se estableció una meta relacionada con actividad física con una disminución importante del porcentaje de población sedentaria (medida en mayores de 15 años, ya que ese dato sólo se registra en ese grupo de población). Las intervenciones en promoción de la salud juegan un papel preponderante en la adquisición de hábitos de vida saludables por los niños ${ }^{9}$. Contar con un diagnóstico de las características de los escolares antes de formular una intervención es fundamental.

Las características antropométricas de la muestra indicaron que la curva de distribución de esta variable está desplazada hacia la derecha de la referencia. Como ésta se basa en niños norteamericanos, en los cuales la proporción de obesos no solamente es alta sino que ha aumentado significativamente en las últimas décadas, este hecho es muy preocupante ${ }^{10}$.

Los resultados referentes al estado nutricional de estos escolares son similares a los informados por Olivares y cols ${ }^{11}$, provenientes de una muestra de escolares también de escuelas municipales

Tabla 4. Estado nutricional y resistencia aeróbica en hombres por edad

\begin{tabular}{|c|c|c|c|c|c|c|}
\hline \multirow[b]{2}{*}{ Edad (a) } & \multicolumn{6}{|c|}{ Estado Nutricional } \\
\hline & $\begin{array}{c}\text { R buena } \\
(\%)\end{array}$ & $\begin{array}{l}\text { Normal } \\
\text { R regular } \\
\quad(\%)\end{array}$ & $\begin{array}{c}\mathrm{R} \text { mala } \\
(\%)\end{array}$ & $\begin{array}{c}\text { R buena } \\
(\%)\end{array}$ & $\begin{array}{c}\text { Sobrepeso }^{1} \\
\text { R regular } \\
(\%)\end{array}$ & $\begin{array}{c}\mathrm{R} \text { mala } \\
(\%)\end{array}$ \\
\hline $10^{*}$ & 29,1 & 30,4 & 40,5 & 9,4 & 37,7 & 52,9 \\
\hline $11^{*}$ & 21,8 & 32,3 & 45,9 & 10,7 & 33,9 & 55,4 \\
\hline $12^{*}$ & 30,9 & 17,1 & 52,0 & 18,0 & 5,1 & 76,9 \\
\hline 13 & 26,9 & 17,1 & 56,0 & 16,9 & 12,7 & 70,4 \\
\hline
\end{tabular}

${ }^{1}$ Incluye sobrepeso y obeso

R Resistencia aeróbica

$*^{2} \mathrm{p}<0,05$

Tabla 5. Estado nutricional y resistencia aeróbica en mujeres por edad

\begin{tabular}{|c|c|c|c|c|c|c|}
\hline \multirow{3}{*}{ Edad (a) } & \multicolumn{6}{|c|}{ Estado Nutricional } \\
\hline & & Normal & & & Sobrepeso $^{1}$ & \\
\hline & $\begin{array}{c}\text { R buena } \\
\text { (\%) }\end{array}$ & $\begin{array}{c}\text { R regular } \\
(\%)\end{array}$ & $\begin{array}{c}\mathrm{R} \text { mala } \\
(\%)\end{array}$ & $\begin{array}{c}\text { R buena } \\
(\%)\end{array}$ & $\begin{array}{c}\mathrm{R} \text { regular } \\
(\%)\end{array}$ & $\begin{array}{c}\mathrm{R} \text { mala } \\
(\%)\end{array}$ \\
\hline $10 *$ & 11,0 & 18,4 & 70,6 & 2,7 & 12,0 & 85,3 \\
\hline $11 *$ & 20,0 & 24,0 & 56,0 & 2,7 & 23,0 & 74,3 \\
\hline $12^{*}$ & 14,5 & 16,8 & 68,7 & 5,7 & 11,5 & 82,8 \\
\hline 13 & 8,8 & 19,6 & 71,6 & 3,5 & 14,0 & 82,5 \\
\hline
\end{tabular}

${ }^{1}$ Incluye sobrepeso y obeso

R Resistencia aeróbica

$*^{*} \chi^{2} \mathrm{p}<0,05$ 
de 3 ciudades del país. En esa muestra (incluye niños de $3^{\circ}$ a $7^{\circ}$ básico), la prevalencia de obesidad (medida con el mismo criterio) en los grupos de 8-9, 10-11 y 12-13 años fue la siguiente: $21,3 \%, 16,2 \%$ y $13,5 \%$ y $15,4 \%, 12,9$ y 11,7 en hombres y mujeres respectivamente. Al igual que en nuestro estudio, en el grupo de menor edad la prevalencia de obesidad fue mayor, encontrándose, además, diferencia por sexo sólo en este grupo, lo que no ocurrió en nuestro caso.

La prevalencia de obesidad en niños chilenos es alta y se ha venido incrementando sostenidamente en los últimos 20 años ${ }^{12}$. Es así como en primer año básico, esta prevalencia se triplicó en 13 años, de 7\% en 1987 a 17,2\% en 2002 (www.junaeb.cl). En este estudio como en el de Olivares, la prevalencia de obesidad fue mayor en los hombres. Bellizzi y cols ${ }^{13}$ que analizaron datos antropométricos en escolares entre 10 y 18 años, de varios países asiáticos y europeos, observaron que la prevalencia de obesidad era mayor en los hombres hasta los 15 años, situación que se revertía en los mayores de esa edad. Nuestros resultados muestran que a partir de los 12 años, la prevalencia de obesidad es igual en ambos sexos.

La prevalencia de sobrepeso fue similar en los grupos de edad estudiados, sin embargo, la obesidad fue mayor en los de menor edad. Este fenómeno podría deberse en parte a la tendencia secular, la obesidad está afectando progresivamente a cohortes más jóvenes. Se podría especular que factores de riesgo de obesidad afectarán progresivamente a niños más pequeños en poblaciones en etapa de transición nutricional. A medida que aumenta el ingreso, el sedentarismo y las dietas ricas en grasas aparecen como factores de riesgo de obesidad en forma más temprana ${ }^{14}$.

En relación al test de Mathews, vale la pena comentar que en la literatura aparece predominantemente otra prueba para determinar la flexibilidad, la de Wells y Dillon adaptada, que se realiza en posición sentada en vez de parada, existiendo una correlación altísima entre ambas pruebas ${ }^{4}$. No fue posible categorizar a los niños según flexibilidad, ya que no consideramos pertinente comparar nuestros resultados con los que obtuvieron Trujillo y cols ${ }^{4}$, único estudio nacional que presenta percentiles de la prueba de Mathews, ya que su muestra incluyó sólo a escolares de un colegio particular. Sin embargo, pudimos verificar que en nuestro estudio, los resultados promedios en todos los grupos de edad y en ambos sexos, fueron significativamente peores que los que obtuvieron Trujillo y cols. Nosotros especulamos que, al provenir la muestra de Trujillo de un colegio particular pagado, esos niños probablemente efectuaban más educación física y de mejor calidad. Sin embargo, este mismo razonamiento no explica totalmente los resultados de la prueba de Navette, ya que en los hombres y mujeres de 10 años y las niñas de 11 años, los valores fueron similares. Lo que sí hay que reconocer es que, en general, las cifras de la prueba de Navette obtenidas por Trujillo, también fueron mejores. Interesante fue la comparación de nuestros datos con los de Castillo y cols, cuya muestra también incluyó a escolares de colegios municipalizados. Se observó que sólo los varones de esa muestra tuvieron una mejor resistencia aeróbica. Se ha demostrado que el nivel socioeconómico es un factor determinante de la actividad física ${ }^{15}$, y que podría existir una asociación entre ésta y lo que generalmente ocurre con escolares de escuelas públicas, quienes tienen menos horas de clases de educación física o menos actividad física fuera del colegio. Aranceta y cols ${ }^{16}$ mostraron que escolares españoles con madres de bajo nivel educacional (proxy de nivel socioeconómico) desarrollaban menor actividad física y miraban más TV. En nuestro país, Godoy ${ }^{17}$ analizó los resultados de estudios sobre capacidad aeróbica en escolares de diferente nivel socioeconómico publicados previos a 1999, concluyendo que existía una asociación directa entre ambas variables. Es decir, la potencia aeróbica aumentaba linealmente con la edad y era mayor en escolares provenientes de colegios particulares. Sin embargo, cuando este mismo investigador ${ }^{18}$ realizó un estudio en 2001, que incluyó a 273.000 estudiantes de 6 a 19 años de escuelas municipalizadas, subvencionadas y particulares de todo el país, mostró que aun cuando se observaba una tendencia a mejores resultados en los colegios particulares, las diferencias no fueron significativas por edad y sexo. El puntualizó, eso sí, que la proporción de alumnos con valores bajo el promedio fue mayor en los alumnos de escuelas municipalizadas.

No cabe duda que la comparación de resultados de distintos estudios es importante, pero mucho más relevante para la promoción de la 
salud, es caracterizar la aptitud física de los escolares chilenos. En ese sentido, los resultados referentes a resistencia aeróbica (prueba de Navette) son tremendamente preocupantes en todos los grupos de edad estudiados y en ambos sexos. Incluso en el grupo con mejores resultados (hombres de 10 años, o sea los menores) hay 40,5\% con resistencia «mala». Esta cifra aumenta sustancialmente en los de 13 años (56\%), lo que muestra que el deterioro es muy rápido. En las niñas, la situación es muchísimo peor y similar por grupo de edad. En ambos sexos, la resistencia aeróbica de los niños con sobrepeso disminuye significativamente.

El test Navette ha sido ampliamente utilizado para determinar la máxima capacidad aeróbica en niños y adultos especialmente en Europa, ya que forma parte del protocolo llamado Eurofit ${ }^{19}$. Manios y cols 20,21 en un estudio con escolares de Creta incluyeron esta prueba para evaluar los efectos de una intervención, observando valores muy similares a los nuestros. Es así como en escolares de aproximadamente 9 años, estos valores fueron de 3,4 períodos en las escuelas intervenidas y 3,2 en las escuelas control.

La asociación entre el IMC y la capacidad aeróbica también ha sido demostrada en otros estudios. Es así como Graf y cols ${ }^{22}$, en un estudio que incluyó a 668 niños de 7 años, encontraron que la habilidad motora gruesa (medida a través

\section{REFERENCIAS}

1. Salnas J, Vio F. Promoción de la Salud en Chile. Rev Chil Nutr 2002; 29: 164-73.

2. Sтову M. School-based approaches for preventing and treating obesity. IJO 1999; 23: S43-S51.

3. MINSAL Consejo Vida Chile y Plan de Promoción de la Salud. Avance de Gestión 2002, Santiago, 2002.

4. Leger LA, Mercier D, Gadoury C, Lambert J. The multistage 20 metre shuttle run test for aerobic fitness. J Sports Sci 1988; 6: 93-101.

5. Mercier D, Leger LA, LAmbert J. Relative efficiency and predicted $\mathrm{VO}_{2}$ max in children. Med Sci Sports Exe 1983; 15: 143-7.

6. Freedson P, Cureton K, Heath G. Status of fieldbased fitness testing in children and youth. Prev Med 2000; 31: S77-S85. de una batería de tests) y la capacidad aeróbica (determinada con la carrera de $6 \mathrm{~min}$ ) fueron significativamente peores en los niños con sobrepeso. Raitakari y cols ${ }^{23}$ mostraron no sólo asociación entre IMC y capacidad aeróbica en niños, sino además con lípidos sanguíneos, siendo este hallazgo mucho más evidente en varones.

En conclusión, los resultados obtenidos del presente estudio muestran la alta prevalencia de obesidad en escolares de educación básica, especialmente en los menores y lo alejada que están estas cifras de la meta propuesta por Vida Chile. Además, se demuestra el deficiente nivel de aptitud física. Estos hallazgos son muy preocupantes, ya que la mayoría de los hábitos de vida se forman en la niñez ${ }^{24}$ e indican la imperiosa necesidad de realizar acciones que impacten estos aspectos. Los resultados de la línea base fueron el eje para centrar la estrategia de intervención en alimentación/nutrición y actividad física que se aplicó durante 6 meses en 2002. En el componente alimentación, se implementó el programa educativo «ducación en nutrición en escuelas básicası ${ }^{25}$ dirigido a escolares de $3^{\circ}$ a $8^{\circ}$ año básico, mientras que en actividad física se aumentó en una hora las clases de educación física y paralelamente se implementó el programa motivacional «esafío Chileno para una Vida Activa» propuesto por el MINEDUC, Chiledeportes $\mathrm{y}$ MINSAL $^{26}$.

7. BARLOW S, DiETZ W. Obesity evaluation and treatment: expert committee recommendations. Pediatrics 1998; 102: E29-E36.

8. MINSAL Unidad de Nutrición. Normas de evaluación del estado nutricional del niño de 6 a 18 años. Documento Interno, 2003.

9. McGraw SA, Seluers D, Stone E. Measuring implementation of school programs and policies to promote healthy eating and physical activity among youth. Prev Med 2000; 31: S86-S97.

10. American Academy of Pediatrics. Policy Statement. Committee on School Health. Pediatrics 2004; 113: 152-4.

11. Olivares S, Kain J, Lera L, Pizarro F, Vio F, Morón C. Nutritional status, food consumption and physical activity among Chilean school children: a descriptive study. Eur J Clin Nutr. 2004; 58: 127885. 
12. Kain J, Uauy R, Vio F, Albala C. Trends in overweight and obesity in Chilean children: comparison of three definitions to determine prevalence. Eur J Clin Nutr 2002: 56; 200-4.

13. Beuzzi M, Horgan G, Gilaume M, Dietz W. Prevalence of childhood obesity and adolescent overweight and obesity in Asian and European countries. En: Obesity in childhood and adolescence. Chen C y Dietz W eds. Nestlé Nutrition Workshop Series Pediatric Program volume 49: pg 23-35, 2001.

14. Kain J, Burrows R, UAuY R. Obesity trends in Chilean children and adolescents: basic determinants. En: Obesity in childhood and adolescence. Chen C y Dietz W eds. Nestlé Nutrition Workshop Series Pediatric Program volume 49: pg 45-62, 2001.

15. FRENN M, MaLn S. Diet and exercise in lowincome culturally diverse middle school students. Public Health Nurs 2003; 20: 361-8.

16. Aranceta J, Pérez-Rodrigo C, Ribas L, Serra-Majem L. Sociodemograghic and lifestyle determinants of food patterns in Spanish children and adolescents: the en Kid Study. Eur J Clin Nutr. 2003; 57: S40-S44.

17. Sociedad MÉdica Educacional Ltda. Estudio para la Elaboración del Plan 2001-2006. Programa Formación para el Deporte Digeder. Informe Final, Agosto 2000.

18. Sociedad Médica Educacional Ltda. Aplicación Batería de Pruebas de Aptitud Física a Población Escolar Nacional 2001. Informe Final presentado a Digeder. Santiago, 2002.
19. Committee of Experts on Sports Research. The Eurofit Test of Physical Fitness: VI ${ }^{\text {th }}$ European Research Seminar, Izmir, June 26-30, 1990.

20. Manios Y, Kafatos A, Mamalakis G. The effects of a health education intervention initiated at first grade over a 3 years period: physical activity and fitness indices. Health Education Research 1998; 13: 593-606.

21. Manios Y, Kafatos A, Codrington C. Gender differences in physical activity and physical fitness in young children in Crete. The Journal of Sports Medicine and Physical Fitness 1999; 39: 24-30.

22. Graf C, Koch B, Kretschmann-Kandel E, FalKowski G, Christ H y cols. Correlation between BMI, leisure habits and motor abilities in childhood (CHILT-Project). IJO 2004; 28: 22-6.

22. Raitakari O, Taimela S, Porkka K, Telama R, VALIMAKI I ET AL. Associations between physical activity and risk factors for coronary heart disease: the Cardiovascular Risk in Young Finns Study. Med Sci Sports Exe 1997; 29: 1055-61.

23. HAYMAN LL, ReINEKe PR. Preventing coronary heart disease: the implementation of healthy lifesytle strategies for children and adolescents. J Cardiovasc Nurs 2003; 18: 294-301.

24. Olivares S, Morón C, Zacarías I, Andrade M, Vio F. Educación en nutrición en las escuelas básicas de Chile. Food Nutr Agr 2003; 33: 64-9.

25. Calc Program. CALC Web Page (http:// www.cahperd.ca/calc/index.html)

Agradecimiento

Los autores agradecen al profesor Héctor Trujillo del MINEDUC, por su apoyo en el análisis de los datos. 